\title{
COVID-19 pandemic-related anxiety, distress and burnout: prevalence and associated factors in healthcare workers of North-West Italy
}

Andrea Naldi, Fabrizio Vallelonga, Alessandra Di Liberto, Roberto Cavallo, Monica Agnesone, Marco Gonella, Maria Domenica Sauta, Piergiorgio Lochner, Giacomo Tondo, Nicola Luigi Bragazzi, Rossana Botto* and Paolo Leombruni*

\section{Background}

The COVID-19 pandemic caused drastic changes in healthcare and severe social restrictions. Healthcare workers (HCWs) are on the front line against the virus and have been highly exposed to pandemic-related stressors, but there are limited data on their psychological involvement for a large sample in Italy.

\section{Aims}

To investigate the prevalence of anxiety, distress and burnout in HCWs of North-West Italy during the COVID-19 pandemic, and to detect potential psychosocial factors associated with their emotional response.

\section{Method}

This cross-sectional, survey-based study enrolled 797 HCWs. Participants completed the Impact of Event Scale - Revised, the State-Trait Anxiety Inventory - Form Y and the Maslach Burnout Inventory; demographic, family and work characteristics were also collected. Global psychological outcome, differences among professions and independent factors associated with worst psychological outcome were assessed.

\section{Results}

Almost a third of the sample had severe state anxiety and distress, high emotional exhaustion and depersonalisation, and low personal accomplishment. Distress was higher in women and nurses, whereas depersonalisation was higher in men. Family division, increased workload, job changes and frequent contact with COVID-19 were associated with worst psychological outcome. Trait anxiety was associated with significantly higher risk for developing state anxiety, distress and burnout.

\section{Conclusions}

An elevated psychological burden related to the COVID-19 pandemic was observed in HCWs of North-West Italy. The identification of family and work characteristics and a psychological pre-existing condition as factors associated with worst psychological outcome may help provide a tailored, preventive, organisational and psychological approach in counteracting the psychological effects of future pandemics.

\section{Keywords}

COVID-19; healthcare workers; anxiety; burnout; Italy.

\section{Copyright and usage}

(c) The Author(s), 2021. Published by Cambridge University Press on behalf of the Royal College of Psychiatrists. This is an Open Access article, distributed under the terms of the Creative Commons Attribution licence (http://creativecommons.org/ licenses/by/4.0/), which permits unrestricted re-use, distribution, and reproduction in any medium, provided the original work is properly cited.
The progressive spread of COVID-19 imposed radical reorganisation in healthcare systems worldwide, to face the ongoing pandemic. Contextually, governments established heavy social restrictions, including quarantine and lockdown, to limit the contagion. The immediate and significant effects of these measures have affected individuals' quality of life in terms of changes to daily and working routine, as well as limiting their social and affective relationships, possibly leading to a reduction in mental well-being. Emotional distress during the COVID-19 pandemic has been related to several stressors, including uncertainty regarding health and social and financial repercussions. ${ }^{2}$ A considerable proportion of the general population have suffered anxiety symptoms and an overall increase in depressive disorders, suicidal ideation and trauma-related symptoms has been documented. ${ }^{3,4}$ Healthcare workers (HCWs) are in the front line against the virus, and are highly exposed to the stressors associated with the pandemic. In this extremely demanding health crisis, they have been asked to multiply their efforts through drastic variations in their usual job duties, potentially resulting in an unexpected psychological burden, particularly burnout. Even if burnout syndrome is often described in this occupational category worldwide, ${ }^{5}$ there are limited data available on Italian HCWs during the COVID-19 pandemic, and available data are for small samples. ${ }^{6,7}$ Studies on the

* Rossana Botto and Paolo Leombruni are equal contributors. psychological impact of previous epidemics on HCWs have shown clinically relevant levels of anxiety, distress and burnout. ${ }^{8-10}$ However, in the modern era, the COVID-19 pandemic is considered to be an unparalleled and incomparable emergency. Preliminary evidence on HCWs exposed to COVID-19 in China and other Eastern countries have confirmed the elevated psychological burden in terms of anxiety, depression, sleep disturbances and post-traumatic symptoms. ${ }^{1-14}$ Nevertheless, although data on the emotional response to the COVID-19 pandemic are increasing, the effects on HCWs of Western nations are understudied and, similarly, little is known about which occupational and socio-familial stressors could have elicited the dysfunctional emotive reactions to the pandemic.

\section{COVID-19: the situation in Italy during April-May 2020}

Northern Italy led the way in the spread of contagion in Europe, and its HCWs were the first in the Western world to experience the dramatic family and occupational rearrangements determined by the pandemic. The first Italian case was isolated on 20 February 2020, and the Italian healthcare system was quickly overwhelmed by the strong rise in positive COVID-19 cases that reached 207,428 on the 1 May 2020, with 28,236 deaths. ${ }^{15}$ The Italian Government imposed quarantine from 10 March to 3 May 2020, corresponding with the highest rate of contagion, hospital admissions and deaths. The implemented restrictive measures were introduced to limit the spread of the virus, and included the suspension of non-essential 
commercial activities, closure of catering business, a ban on public gatherings, severe limitations to interregional travelling and closure of educational, sociocultural and religious activities. ${ }^{16}$ Despite these restrictions, the progressive and continuous increase in patients with COVID-19 led to the saturation of intensive care units beds and emergency departments in Italian hospitals, followed by significant structural changes and the reorganisation of medical and surgical wards for treatment of patients with COVID-19. As a result, HCWs faced an unexpected increase in workload, busy shifts and changes in their working routine that, combined with the absence of clear guidelines and protocols for the management of the infection, led them to physical exhaustion and feelings of uncertainty, loneliness and alienation. ${ }^{6}$ In addition, the initial unavailability of personal protective equipment and the unpreparedness of the Italian healthcare system massively increased HCWs' vulnerability and, by 9 April 2020, more than 10,000 cases among HCWs (predominantly in the Northern regions) had been recorded. ${ }^{17}$ Because of direct exposure to potentially infected patients, fear of virus transmission was high, as was the fear of endangering relatives at home. Thus, the use of protective masks was also highly recommended in personal contexts, sometimes determining family division of HCWs to reduce the risk of contagion. All of these factors may have contributed to the development of psychological symptoms in HCWs.

Considering the evidence and the limited data available for Italian HCWs in this area, the aim of this study was to investigate the prevalence of anxiety, distress and burnout in a large sample of physicians and nurses working in Turin city, Piedmont, Northern Italy, during the COVID-19 pandemic, and to detect potential psychosocial family- and work-related factors potentially associated with their emotional response.

\section{Method}

\section{Study design and participants}

This cross-sectional, survey-based study recruited HCWs from four hospitals in Turin city, Piedmont, Northern Italy. The hospitals involved were all facing the pandemic and were active in the management of patients with COVID-19, covering a population area of about 870,000 inhabitants. All procedures contributing to this work comply with the ethical standards of the relevant national and institutional committees on human experimentation and with the Helsinki Declaration of 1975 , as revised in 2008. The study received ethical approval by the local institutional review board (A.S.L. Città di Torino). The study was performed according to the Strengthening the Reporting of Observational Studies in Epidemiology Statement (Supplementary material available at https://doi.org/10.1192/bjo.2020.161).

We chose an online survey to collect the data. The online survey allowed for social distancing to be ensured and the elimination of interpersonal interaction, as required by the Italian Government. Before beginning, on the first page of the questionnaire, participants were informed on the scope of the study and their voluntary participation in the survey, which was executed in accordance with current legislation regarding ethical standards and privacy protection. Participants were asked to confirm that they agreed with the information provided, were aged $\geq 18$ years and consented to participation online by selecting the term 'I agree'. The questionnaire was anonymous and electronic consent was obtained from all participants before taking part. The survey was sent by email simultaneously to all physicians and nurses of the referring hospitals on 27 April 2020, explaining that enrolment would end automatically upon achievement of the target sample size. To create the survey, we used the free platform Google Forms 2020
(Google Inc., USA; see https://www.google.it/intl/it/forms/about/). A minimum sample size of 377 participants was estimated, using the formula $N=Z^{2} \times p(1-p) / \alpha^{2}$, considering a margin of error of $5 \%(\alpha=0.05)$, a $95 \%$ confidence interval $(Z=1.96)$ and a population proportion of $50 \%(P=0.50)$. To favour subgroup analysis, the number of participants was at least doubled. After reaching the target number, recruitment was interrupted at midnight of that day (1 May 2020). These 5 days over which the survey was open were during the more demanding period of the COVID-19 pandemic in Italy (10 March to 3 May 2020). At survey closure, a total of 797 respondents had completed the questionnaire.

\section{Survey characteristics}

The survey investigated the demographic, family and work characteristics of the recruited HCWs, and participants were asked to complete a set of validated rating scales to assess state and trait anxiety, distress and occupational burnout.

Sociodemographic data included age range, gender, job position and the result of a COVID-19 swab test. Ad hoc questions on the family situation explored the following areas: having children, current living situation (with partner, without partner) and family division owing to the pandemic (i.e. living separated from own family for safety reasons, fear of endangering relatives). Work queries included contact frequency with patients with COVID-19 patients ('frequent' if exposure to infected patients was continuous or almost continuous, 'rare' if it was only occasional or absent), occurrence of job changes related to the emergency (i.e. relevant modifications in usual job duties and activities as a result of the pandemic) and variations in workload (increased or not increased, based on the subjective opinion of the HCW).

\section{Tools}

Italian validations of the following three rating scales were used. The Impact of Event Scale - Revised (IES-R) $)^{18,19}$ is a self-report scale widely used to measure current subjective distress related to a specific stressful life event, including outbreaks. ${ }^{20}$ It consists of 22 items on a 5-point Likert scale (from 0 , 'not at all', to 4 , 'extremely'), with respect to how distressing each item has been during the past week. The IES-R assesses symptoms of avoidance, intrusion and hyperarousal. The total score ranges from 0 to 88 . The scores identify four levels of distress symptoms: normal, indicating the absence of post-traumatic symptoms (0-23); mild, indicating clinically detectable post-traumatic symptoms (24-32); moderate, indicating the possible presence of post-traumatic stress disorder (33-36); and severe, indicating severe post-traumatic symptoms $(\geq 37) .{ }^{18}$

The State-Trait Anxiety Inventory - Form Y (STAI-Y) ${ }^{21,22}$ is one of the most widely used self-reported measures of anxiety. ${ }^{23}$ It is a self-report inventory aimed at assessing both state (STAIY1) and trait (STAI-Y2) anxiety, consisting of 40 items on a 4point Likert scale (from 1, 'almost never', to 4 , 'almost always'). ${ }^{22}$ State anxiety refers to 'subjective feelings of tension, apprehension, nervousness, and worry, and activation or arousal of the autonomic nervous system, which exist at a given moment in time and at a particular level of intensity' (page 4). ${ }^{22}$ Trait anxiety refers to 'relatively stable individual differences in anxiety-proneness, that is, to differences between people in the tendency to perceive stressful situation as dangerous or threatening and to respond to such situations with elevations in the intensity of their state anxiety reactions' (page 5). ${ }^{22}$ Twenty items are dedicated to each anxiety type. Scores for both subscales range between 20 and 80 . For both subscales, scores of $40-50$ indicate mild anxiety, 50-60 indicate moderate anxiety and $>60$ indicate severe anxiety. ${ }^{22}$

The Maslach Burnout Inventory (MBI) ${ }^{24,25}$ is the most common instrument used to measure occupational burnout. ${ }^{26}$ The 


\begin{tabular}{|c|c|c|c|c|}
\hline \multirow[b]{2}{*}{ Characteristic } & \multirow[b]{2}{*}{ Overall $(n=797)$} & \multicolumn{2}{|c|}{ Healthcare workers } & \multirow[b]{2}{*}{$P$-value } \\
\hline & & Physician $(n=328)$ & Nurse $(n=469)$ & \\
\hline \multicolumn{5}{|l|}{ Gender, $n(\%)$} \\
\hline Male & $198(24.8)$ & $122(37.2)$ & $76(16.2)$ & \multirow[t]{2}{*}{$<0.01$} \\
\hline Female & $599(75.2)$ & $206(62.8)$ & $393(83.8)$ & \\
\hline \multicolumn{5}{|l|}{ Age, years, $n$ (\%) } \\
\hline $18-30$ & $90(11.3)$ & $18(5.5)$ & $72(15.4)$ & \multirow{4}{*}{$\begin{array}{l}<0.01 \\
\text { Not significant } \\
\text { Not significant } \\
<0.01\end{array}$} \\
\hline $31-40$ & $179(22.5)$ & $83(25.3)$ & $96(20.5)$ & \\
\hline $41-50$ & 248 (31.1) & $90(27.4)$ & $158(33.7)$ & \\
\hline$>50$ & $280(35.1)$ & $137(41.8)$ & $143(30.5)$ & \\
\hline \multicolumn{5}{|c|}{ COVID-19 test, $n(\%)$} \\
\hline Negative & $711(89.2)$ & 300 (91.5) & $411(87.6)$ & \multirow[t]{2}{*}{ Not significant } \\
\hline Positive & $86(10.8)$ & $28(8.5)$ & $58(12.4)$ & \\
\hline \multicolumn{5}{|l|}{ Children, $n$ (\%) } \\
\hline No & $291(36.5)$ & $108(32.9)$ & 183 (39) & \multirow[t]{2}{*}{ Not significant } \\
\hline Yes & $506(63.5)$ & $220(67.1)$ & $286(61)$ & \\
\hline \multicolumn{5}{|c|}{ Living condition, $n$ (\%) } \\
\hline Without partner & $233(29.2)$ & $74(22.6)$ & $159(33.9)$ & \multirow[t]{2}{*}{$<0.01$} \\
\hline With partner & $564(70.8)$ & $254(77.4)$ & $310(66.1)$ & \\
\hline \multicolumn{5}{|c|}{ Family division, $n$ (\%) } \\
\hline No & $591(74.2)$ & $232(70.7)$ & 359 (76.5) & \multirow[t]{2}{*}{ Not significant } \\
\hline Yes & $206(25.8)$ & $96(29.3)$ & $110(23.5)$ & \\
\hline \multicolumn{5}{|c|}{ COVID-19 contact, $n$ (\%) } \\
\hline Rare & $234(29.4)$ & 99 (30.2) & $135(28.8)$ & \multirow[t]{2}{*}{ Not significant } \\
\hline Frequent & $563(70.6)$ & $229(69.8)$ & $334(71.2)$ & \\
\hline \multicolumn{5}{|l|}{ Job changes, $n$ (\%) } \\
\hline No & 349 (43.8) & $140(42.7)$ & 209 (44.6) & \multirow[t]{2}{*}{ Not significant } \\
\hline Yes & $448(56.2)$ & 188 (57.3) & $260(55.4)$ & \\
\hline \multicolumn{5}{|l|}{ Workload, $n$ (\%) } \\
\hline Not increased & $322(40.4)$ & $164(50)$ & $158(33.7)$ & \multirow[t]{2}{*}{$<0.01$} \\
\hline Increased & 475 (59.6) & $164(50)$ & $311(66.3)$ & \\
\hline
\end{tabular}

questionnaire is composed by three scales: emotional exhaustion (nine items), examining the feeling of being emotionally bitter and exhausted from work; depersonalisation (five items), which measures a cold and impersonal response; and personal accomplishment (eight items), assessing the feeling of one's own competence and desire to succeed in working with others. High scores on the personal accomplishment scale, unlike previous emotional exhaustion and depersonalisation scales, indicates greater personal achievement and, consequently, a lower level of burnout. All of the MBI items are scored with a 7-point Likert scale (from 1, 'never', to 7, 'daily'). Consistent with the literature, we considered the level of burnout to be high if emotional exhaustion scores were $\geq 24$, personal accomplishment scores were $\leq 29$ and depersonalisation scores were $\geq 9$; moderate if emotional exhaustion scores were 15-23, personal accomplishment scores were 30-36 and depersonalisation scores were 4-8; and low if emotional exhaustion scores were $\leq 14$, personal accomplishment scores were $\geq 37$ and depersonalisation scores were $\leq 3 .^{27}$

\section{Statistical analysis}

The normal distribution of variables was tested with KolmogorovSmirnov and residual analysis tests. Continuous variables were expressed as mean \pm s.d. or median and interquartile range, as appropriate. Qualitative variables were expressed as absolute values of frequency and percentage values. Differences between independent groups were evaluated by a $t$-test for continuous variables with normal distribution, and the Mann-Whitney $U$ or Kruskal-Wallis test for continuous variables with non-normal distribution. Categorical variables were compared with the chi-square test or Fisher's exact test, as appropriate. A multivariable logistic regression was applied, using the presence of moderate or severe symptoms (for each psychological scale) as a dependent variable and possible risk factors as independent variables. The associations between risk factors and psychological symptoms are presented as odds ratios and $95 \%$ confidence intervals. Statistical significance was considered for $P$-values $<0.05$ in all analyses. Statistical analysis was performed with software package SPSS (IBM SPSS Statistics for Windows, version 22; IBM Corp., New York, USA).

\section{Results}

\section{Participants}

The study sample was composed of 797 participants ( $75.2 \%$ female); 328 (41.2\%) were physicians and $469(58.8 \%)$ were nurses. The majority of the participants $(n=528,66.2 \%)$ were aged over 40 years and $563(70.6 \%)$ had frequent contact with patients with COVID-19. A detailed description of the demographics and family and working conditions for both physicians and nurses is provided in Table 1.

\section{Psychological outcomes}

A total of 618 (77.5\%) participants had state anxiety and 478 (60\%) reported distress symptoms such as avoidance, intrusion and hyperarousal. A considerable proportion of these HCWs suffered severe symptoms, both in terms of state anxiety $(n=186,23.3 \%)$ and distress $(n=286,35.9 \%)$.

Regarding the three dimensions of occupational burnout, 324 (40.7\%) HCWs showed high emotional exhaustion, 241 (30.2\%) displayed high depersonalisation and 290 (36.4\%) had low personal accomplishment (see Table 2 for full details). Data on trait anxiety, including scores and levels, are reported in the Supplementary Material.

By stratifying for symptom severity levels, no differences were found between physicians and nurses for either anxiety or occupational burnout. Conversely, nurses showed a higher prevalence of 
Table 2 Psychological outcomes stratified for scores and severity categories

\begin{tabular}{|c|c|c|c|c|c|}
\hline \multirow{2}{*}{ Psychological scale } & & \multirow[b]{2}{*}{ Overall $(n=797)$} & \multicolumn{2}{|c|}{ Healthcare workers } & \multirow[b]{2}{*}{$P$-value } \\
\hline & & & Physician $(n=328)$ & Nurse $(n=469)$ & \\
\hline \multicolumn{6}{|l|}{ STAI Y1 } \\
\hline \multicolumn{2}{|l|}{ Total scoring, median (IQR) } & $50(41-59)$ & $49(40-58)$ & $50(41-60)$ & Not significant \\
\hline \multirow[t]{4}{*}{ Categories, $n(\%)$} & Normal & $179(22.5)$ & $74(22.6)$ & 105 (22.4) & Not significant \\
\hline & Mild & $218(27.4)$ & $92(28)$ & $126(26.9)$ & \\
\hline & Moderate & $214(26.9)$ & $98(29.9)$ & $116(24.7)$ & \\
\hline & Severe & $186(23.3)$ & $64(19.5)$ & $122(26)$ & \\
\hline \multicolumn{6}{|l|}{ IES-R } \\
\hline \multicolumn{2}{|l|}{ Total scoring, median (IQR) } & $29(17-44)$ & $26(15-41)$ & $31(20-46)$ & 0.01 \\
\hline \multicolumn{2}{|l|}{ Hyperarousal } & $1.29(0.7-1.9)$ & $1.14(0.7-1.9)$ & $1.29(0.7-2)$ & Not significant \\
\hline \multicolumn{2}{|l|}{ Avoidance } & $0.75(0.3-1.5)$ & $0.75(0.3-1.5)$ & $1(0.5-1.5)$ & $<0.01$ \\
\hline \multicolumn{2}{|l|}{ Intrusion } & $1.75(1-2.5)$ & $1.5(1-2.3)$ & $2(1.3-2.8)$ & $<0.01$ \\
\hline \multirow[t]{4}{*}{ Categories, $n(\%)$} & Normal & $319(40)$ & $151(46)^{*}$ & $168(35.8)^{*}$ & 0.03 \\
\hline & Mild & $128(16.1)$ & $49(14.9)$ & $79(16.8)$ & \\
\hline & Moderate & $64(8)$ & $25(7.6)$ & $39(8.3)$ & \\
\hline & Severe & 286 (35.9) & $103(31.3)^{\star}$ & $183(39)^{*}$ & \\
\hline \multicolumn{6}{|l|}{ MBI - emotional exhaustion } \\
\hline \multicolumn{2}{|l|}{ Total scoring, median (IQR) } & $20(10-31)$ & $20(10-33)$ & $19(10-30)$ & Not significant \\
\hline \multirow[t]{3}{*}{ Categories, $n(\%)$} & Low & $297(37.3)$ & $121(36.9)$ & $176(37.5)$ & Not significant \\
\hline & Medium & $176(22.1)$ & $70(21.3)$ & $106(22.6)$ & \\
\hline & High & $324(40.7)$ & $137(41.8)$ & $187(39.9)$ & \\
\hline \multicolumn{6}{|l|}{ MBI - depersonalisation } \\
\hline \multicolumn{2}{|l|}{ Total scoring, median (IQR) } & $5(1-10)$ & $5(2-10)$ & $4(1-10)$ & Not significant \\
\hline \multirow[t]{3}{*}{ Categories, $n(\%)$} & Low & $346(43.4)$ & 128 (39) & $218(46.5)$ & Not significant \\
\hline & Medium & $210(26.3)$ & $93(28.4)$ & $117(24.9)$ & \\
\hline & High & $241(30.2)$ & $107(32.6)$ & $134(28.6)$ & \\
\hline \multicolumn{6}{|l|}{ MBI - personal accomplishment } \\
\hline \multicolumn{2}{|l|}{ Total scoring, median (IQR) } & $34(26-39)$ & $34(26-39)$ & $34(26-40)$ & Not significant \\
\hline \multirow[t]{3}{*}{ Categories, $n(\%)$} & Low & $290(36.4)$ & $113(34.5)$ & $177(37.7)$ & Not significant \\
\hline & Medium & $222(27.9)$ & $98(29.9)$ & $124(26.4)$ & \\
\hline & High & $285(35.8)$ & $117(35.7)$ & $168(35.8)$ & \\
\hline
\end{tabular}

severe distress than physicians (183 [39\%] v. 103 [31.3\%], $P=0.03$; see Table 2).

\section{Factors associated with burnout, anxiety and distress}

A complete summary of how the demographic, family and occupational factors are associated with each psychological scale is provided in Tables 3 and 4 . With regard to gender, women reported higher levels of severe state anxiety and distress than men $(26.2 \%$ v. $14.6 \%, P<0.01$ and $37.9 \%$ v. $29.8 \%, P=0.01$, respectively), whereas men had a higher level of depersonalisation than women (38.9\% v. $27.4 \%, P<0.01$ ).

After correcting for confounders, multivariable logistic regression analysis showed that family division and increased workload were both independently associated with moderate-to-severe symptoms of state anxiety (odds ratio 1.96, 95\% CI 1.3-2.9, $P<0.01$; and odds ratio $2.9,95 \%$ CI $2-4.2, P<0.01$, respectively), distress (odds ratio $2.14,95 \%$ CI $1.5-3.1, P<0.01$; and odds ratio $2.35,95 \% \mathrm{CI}$ 1.7-3.3, $P<0.01$ ) and emotional exhaustion (odds ratio 1.83 , 95\% CI 1.3-2.7, $P<0.01$; and odds ratio $2.11,95 \%$ CI 1.5-2.9, $P<$ 0.01 ), as described in Table 5. Job changes were related with more moderate-to-severe symptoms of distress and emotional exhaustion (odds ratio $1.63,95 \%$ CI $1.2-2.3, P<0.01$; and odds ratio 1.41 , $95 \%$ CI $1.1-1.9, P=0.03$, respectively), whereas depersonalisation was higher for frequent contact with patients with COVID-19 (odds ratio $2.05,95 \%$ CI $1.5-2.9, P<0.01$ ). Finally, HCWs presenting with trait anxiety was strongly associated with moderate-tosevere symptoms of state anxiety (odds ratio 8, 95\% CI 5.7-11.3, $P<0.01$ ) and distress (odds ratio 5.79, 95\% CI 4.1-8.2, $P<0.01$ ), but medium-to-high symptoms of emotional exhaustion (odds ratio 3.32, 95\% CI 2.4-4.6, $P<0.01$ ) and depersonalisation (odds ratio $2.44,95 \% \mathrm{CI} 1.8-3.3, P<0.01)$. Trait anxiety was also associated with lower risk of having medium-to-low personal accomplishment (odds ratio $0.51,95 \%$ CI $0.4-0.7, P<0.01$ ); after severity stratification, this relationship was only present for mild and moderate levels of trait anxiety (mild: odds ratio 0.51 , $95 \%$ CI $0.4-0.7, P<0.01$; moderate: odds ratio $0.50,95 \%$ CI $0.3-0.8$, $P<0.01)$

\section{Discussion}

This work provides reliable data on the psychological impact of the COVID-19 pandemic in a large sample of HCWs in North-West Italy. Results show a high prevalence of state anxiety, distress and burnout, particularly in their moderate-to-severe forms, and their strict association with the family- and work-related conditions induced by the pandemic. In this context, only limited data in small samples of Italian HCWs are available, highlighting the relevance of our findings. ${ }^{6,7}$

Regarding the prevalence of anxiety and distress, our findings are in line with those reported in the Asian populations for the COVID-19 pandemic and previous descriptions for severe acute respiratory syndrome (SARS) and Middle East respiratory syndrome (MERS), where a massive impact of these outbreaks on HCWs has been described. ${ }^{8,10-12,28}$

Nevertheless, the presented data are consistent because they refer to the first European nation overwhelmed by the contagion. On 1 May - the date of survey closure - Italy was the most compromised European country in terms of deaths, ${ }^{29}$ and Piedmont was the second region for the number of cases, ${ }^{15}$ confirming the elevated psychological burden for HCWs even in a different sociocultural environment with different healthcare systems. 


\begin{tabular}{|c|c|c|c|c|c|c|c|c|c|c|}
\hline Characteristic & \multicolumn{4}{|c|}{ STAI Y1 (category) } & \multicolumn{6}{|c|}{ IES-R (category) } \\
\hline \multicolumn{11}{|l|}{$\begin{array}{l}\text { Overall, } n \text { (\%) } \\
\text { Profession, } n \text { (\%) }\end{array}$} \\
\hline Profession, n (\%) & & & & & & & & & & \multirow{3}{*}{0.03} \\
\hline Physician & 74 (22.6) & $92(28)$ & $98(29.9)$ & 64 (19.5) & \multirow{2}{*}{ Not significant } & $151(46)^{\star}$ & $49(14.9)$ & $25(7.6)$ & $103(31.4)^{*}$ & \\
\hline \multicolumn{6}{|l|}{ Gender, $n$ (\%) } & & $79(16.8)$ & $39(8.3)$ & $183(39)^{*}$ & \\
\hline Male & $63(31.8)^{*}$ & $60(30.3)$ & $46(23.2)$ & $29(14.6)^{*}$ & \multirow[t]{2}{*}{$<0.01$} & $100(50.5)^{\star}$ & $28(14.1)$ & $11(5.6)$ & $59(29.8)^{*}$ & \multirow[t]{2}{*}{0.01} \\
\hline \multicolumn{7}{|l|}{ CovID-19 test, $n(\%)$} & & $53(8.8)$ & $227(37.9)^{*}$ & \\
\hline Negative & $156(21.9)$ & $202(28.4)$ & $193(27.1)$ & $160(22.5)$ & \multirow[t]{2}{*}{ Not significant } & $290(40.8)$ & 109 (15.3) & $57(8)$ & $255(35.9)$ & \multirow[t]{2}{*}{ Not significant } \\
\hline \multicolumn{9}{|l|}{ Children, $n$ (\%) } & & \\
\hline Yes & $110(21.7)$ & $143(28.3)$ & $145(28.7)$ & $108(21.3)$ & \multirow[t]{2}{*}{ Not significant } & $205(40.5)$ & $74(14.6)$ & $41(8.1)$ & $186(36.8)$ & \multirow[t]{2}{*}{ Not significant } \\
\hline \multicolumn{9}{|l|}{ Living condition, $n$ (\%) } & & \\
\hline With partner & $129(22.9)$ & $159(28.2)$ & $149(26.4)$ & $127(22.5)$ & \multirow[t]{2}{*}{ Not significant } & $226(40.1)$ & $90(16)$ & $41(7.3)$ & $207(36.7)$ & \multirow{2}{*}{ Not significant } \\
\hline \multicolumn{9}{|l|}{ Family division, $n$ (\%) } & & \\
\hline Yes & $30(14.6)^{\star}$ & $48(23.3)$ & $76(36.9)^{*}$ & $52(25.2)$ & \multirow[t]{2}{*}{$<0.01$} & $49(23.8)^{\star}$ & $37(18)$ & $15(7.3)$ & $105(51)^{\star}$ & \multirow[t]{2}{*}{$<0.01$} \\
\hline \multicolumn{9}{|l|}{ CovID-19 contact, $n(\%)$} & & \\
\hline Rare & $61(26.1)$ & $65(27.8)$ & $53(22.6)$ & $55(23.5)$ & \multirow[t]{2}{*}{ Not significant } & $123(52.6)^{*}$ & $34(14.5)$ & $17(7.3)$ & $60(25.6)^{\star}$ & \multirow[t]{2}{*}{$<0.01$} \\
\hline $\begin{array}{l}\text { Frequent } \\
\text { Job changes, } n(\%)\end{array}$ & $118(21)$ & $153(27.2)$ & $161(28.6)$ & 131 (23.3) & & $196(34.8)^{\star}$ & $94(16.7)$ & $47(8.3)$ & $226(40.1)^{\star}$ & \\
\hline No & $93(26.6)$ & $95(27.2)$ & $89(35.5)$ & $72(20.6)$ & \multirow[t]{2}{*}{ Not significant } & $153(43.8)$ & $67(19.2)$ & $24(6.9)$ & $105(30.1)^{\star}$ & \multirow{3}{*}{0.01} \\
\hline Yes & $86(19.2)$ & $123(27.5)$ & $125(27.9)$ & $114(25.4)$ & & $166(37.1)$ & $61(13.6)$ & $40(8.9)$ & $181(40.4)^{\star}$ & \\
\hline \multicolumn{10}{|l|}{ Workload, $n$ (\%) } & \\
\hline Increased & $77(16.2)^{\star}$ & $118(24.8)$ & $148(31.2)^{\star}$ & $132(27.8)^{\star}$ & \multirow[t]{2}{*}{$<0.01$} & $144(30.3)^{\star}$ & $79(16.6)$ & $39(8.2)$ & $213(44.8)^{*}$ & \multirow[t]{2}{*}{$<0.01$} \\
\hline Not increased & $102(31.7)^{*}$ & $100(31.1)$ & $66(20.5)^{\star}$ & $54(16.8)^{\star}$ & & $175(54.3)^{\star}$ & $49(15.2)$ & $25(7.8)$ & $73(22.7)^{\star}$ & \\
\hline $\begin{array}{l}\text { STAI Y1, State-Trait Anxiety In } \\
\text { * Statistically significant. }\end{array}$ & ory Form Y1; & of Event S & & & & & & & & \\
\hline
\end{tabular}




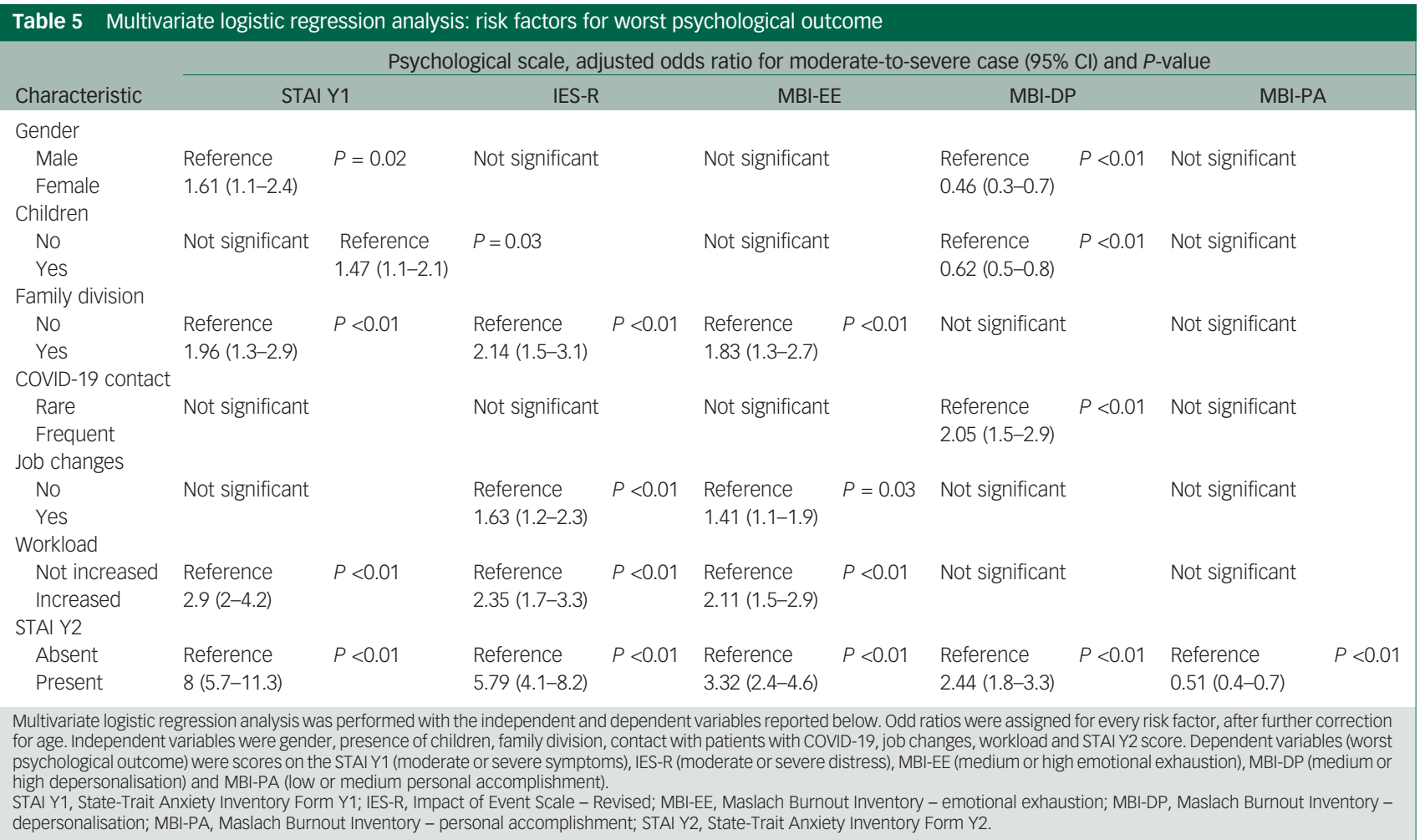

HCWs are subject to developing burnout syndrome, and different levels of emotional exhaustion, depersonalisation and personal accomplishment have been reported. ${ }^{5}$ Average levels of emotional exhaustion and depersonalisation are described in Italian HCWs in their usual working conditions; ${ }^{30}$ however, the baseline levels of burnout depend on the characteristics of the target sample, as well as on the size of the sample and the subjects enrolled in the sample. ${ }^{31-33}$ Nevertheless, the extent of the impact of the COVID19 pandemic on HCWs' burnout levels is still unclear, requiring further studies with larger samples. Preliminary evidence reported a high prevalence of burnout in HCWs during the pandemic, at around $50 \%$ in the three dimensions of burnout of emotional exhaustion, depersonalisation and personal accomplishment in several studies. ${ }^{34,35}$ Our findings confirm that moderate-to-severe levels of burnout are detectable in Italian HCWs facing the COVID-19 pandemic; contextually, they appear higher when referring to the normative values. ${ }^{23}$

Among professions, in line with previous evidence, nurses were more prone to develop severe distress compared with physicians. ${ }^{12,36}$ This finding was specifically driven by higher scores in avoidance and intrusion on the IES-R subscales in our sample. The connection between nurses and distress could be related to a higher susceptibility to infection because of the closer and more continuous relationship with patients. Thus, nurses' vulnerability should be considered when implementing preventive measures, so as to protect them from psychological damage during a pandemic. ${ }^{37}$

Regarding gender, women appeared to be more prone to develop symptoms related to anxiety and distress, whereas depersonalisation was more frequently associated with men (odds ratio 2.17, 95\% CI 1.5-3.2, $P<0.01$ ). Even if gender differences in burnout are uncertain - particularly in a pandemic context where limited data are available - a tendency for major depersonalisation in men has been previously described. ${ }^{38}$ However, further investigation is needed to confirm this finding.

In addition, our findings suggest that division of the family nucleus during the pandemic was associated with worst outcome for developing distress symptoms and emotional exhaustion.
Participants who decided to live at home but separated from loved ones experienced more psychological symptoms, probably because the socio-familial dimension has a relevant role in sustaining an individual's quality of life, and it is known that social support (i.e. sharing feelings, discussing choices and searching for help when needed) is an important resource for psycho-physical health. ${ }^{39}$ Moreover, evidence that family support reduces the risk of mental health issues is well established. ${ }^{40}$ Social isolation can exacerbate the management of distress; ${ }^{41}$ therefore, the sociofamilial dimension of HCWs' quality of life could play a considerable role in their mental health.

With regard to work-related factors, the increased workload, having frequent contact with patients with COVID-19 and changes in job duties were associated with more severe distress and emotional exhaustion both in physicians and nurses, in line with previous reports. ${ }^{6,11,12}$ In a critical condition in which healthcare systems are at risk of collapsing, as with the COVID-19 pandemic, these features could have exacerbated the risk of developing occupational burnout. However, interestingly, the same occupational factors were also associated with significantly higher levels of elevated personal accomplishment (Tables 3 and 4). We suggest that despite the adverse psychological conditions, these job-related difficulties could have increased HCWs' personal satisfaction and sense of fulfilment, especially when associated with contributing toward the health of the community in terms of saving lives and limiting the negative effects of the crisis. Moreover, HCWs have been defined by the social media as 'heroes' for their dedication and work. ${ }^{42}$ As a result, their sense of social responsibility could have been amplified by this attribution of importance to their profession. ${ }^{43}$

Our data show that trait anxiety was strongly associated with state anxiety, distress and burnout during the pandemic. However, HCWs with mild-to-moderate levels of trait anxiety also demonstrated a significantly lower probability of having low personal accomplishment. Anxiety represents a warning reaction to external stimulations, and in its normal state, it enhances the ability of the individual to resolve specific situations. This is 
particularly true when its levels remain subthreshold; in contrast, beyond its threshold, anxiety becomes harmful. It has been postulated that negative emotions such as anxiety could be beneficial for performance; indeed, this so-called 'anxiety motivation' could have facilitative properties in specific circumstances. ${ }^{44}$ Even if the pandemic context generated unfavourable working and family conditions, it could also have provided determination and dynamism, helping to reach a future target. This functional utility of anxiety could have led to higher accomplishment, irrespective of the high levels of distress and emotional exhaustion.

In conclusion, our work provides consistent data on the psychological impact of exposure to COVID-19 and stress-associated factors on HCWs in North-West Italy. Clinically significant symptoms of anxiety, distress and burnout emerged in the examined sample. Nurses and HCWs who experienced family division, increased workload, job changes and trait anxiety were most at risk of developing such symptoms, with some gender differences. These findings are relevant in helping to identify individuals at higher risk of developing psychological concerns during a pandemicrelated health crisis. Based on previous research and findings from this study, protective interventions dedicated to HCWs (i.e. providing valid instrumental support, guaranteeing an adequate jobrelated turnover, using clear operative protocols and making psychological support services available) are needed for protection from clinically relevant symptoms. In this regard, implementing individualised paths to enhance HCWs' resilience could be useful for reducing burnout levels. ${ }^{45}$ The detection of factors associated with worst psychological outcome may favour a tailored, preventive, organisational and psychological approach, representing a potential strength in counteracting the effects of future pandemics.

\section{Limitations}

This study has some limitations. First, we collected data from a large sample of HCWs in Turin, Italy, but the results are specific to the involved area and may not necessarily be generalisable to other healthcare systems or territories with a different pandemic situation. Moreover, among HCWs who had frequent contact with patients with COVID-19, we were unable to stratify them by the level of intensity of care. Second, we recruited only some of the healthcare professionals of the hospitals involved, leading to potential selection bias. Third, the investigation was conceived as cross-sectional and survey-based, thus offering a static snapshot of a pandemic that is, in contrast, an evolving and dynamic situation. Because of its design, longitudinal follow-up is lacking. Finally, the psychological scales used were self-reported and not directly assessed by a mental health professional.

\footnotetext{
Andrea Naldi (D), Rita Levi Montalcini Department of Neuroscience, University of Turin Italy; and Neurology Unit, San Giovanni Bosco Hospital, Italy; Fabrizio Vallelonga, Department of Emergency Medicine, San Giovanni Bosco Hospital, Italy; Alessandra Di Liberto, Neurology Unit, San Giovanni Bosco Hospital, Italy; Roberto Cavallo. Neurology Unit, San Giovanni Bosco Hospital, Italy; Monica Agnesone, Psychology Unit, Local Health Authority of the City of Turin, Italy; Marco Gonella, Psychology Unit, Loca Health Authority of the City of Turin, Italy; Maria Domenica Sauta, Department of Psychology, University of Turin, Italy; Piergiorgio Lochner, Department of Neurology, Saarland University Medical Center, Germany; Giacomo Tondo, School of Psychology, Vita-Salute San Raffaele University, Italy; Nicola Luigi Bragazzi, Department of Vita-Salute San Raffaele University, Italy; Nicola Luigi Bragazzi, Department of
Mathematics and Statistics, Laboratory for Industrial and Applied Mathematics, York University, Ontario, Canada; Rossana Botto, Department of Neuroscience, University of Turin, Italy; and Clinical Psychology Unit, City of Health and Science University Hospital of Turin, Italy; Paolo Leombruni, Department of Neuroscience, University of Turin, Italy; and Clinical Psychology Unit, City of Health and Science University Hospital of Turin, Italy

Correspondence: Andrea Naldi. Email: naldi.andrea@yahoo.it

First received 8 Sep 2020, final revision 20 Nov 2020, accepted 3 Dec 2020
}

\section{Supplementary Material}

Supplementary material is available online at https://doi.org/10.1192/bjo.2020.161.

\section{Data availability}

The data supporting the findings of this study are available from the corresponding author, A.N., upon reasonable request.

\section{Acknowledgements}

We are grateful to all of the survey participants. We thank all our colleagues at the S.S.S. Psicologia Aziendale, A.S.L. Città di Torino and the Neurology Unit of San Giovanni Bosco Hospital for their contributions and cooperation.

\section{Author contributions}

A.N. conceived and designed the study, coordinated data collection, analysed and interpreted the data, participated in the statistical analysis and drafted the manuscript. F.V. performed the statistical analyses and drafted the manuscript. A.D.L., M.A., M.G. and M.D.S. collected and interpreted the data, and participated in the draft of the manuscript. R.C., P. Lochner, G.T. and N.L.B. revised the manuscript, adding important intellectual content. R.B. conceived the study, analysed and interpreted data, and drafted and revised the manuscript. P. Leombruni interpreted the data and revised the manuscript, adding important intellectual content. All authors read and approved the final version of the manuscript.

\section{Funding}

This research received no specific grant from any funding agency, commercial or not-for-profit sectors.

\section{Declaration of interest}

None.

\section{References}

1 Brooks SK, Webster RK, Smith LE, Woodland L, Wessely S, Greenberg N, et al. The psychological impact of quarantine and how to reduce it: rapid review of the evidence. Lancet 2020; 395(10227): 912-20.

2 Pfefferbaum B, North CS. Mental health and the Covid-19 pandemic. N Engl J Med 2020; 383(6): 510-12.

3 da Silva ML, Rocha RSB, Buheji M, Jahrami H, Cunha KdC. A systematic review of the prevalence of anxiety symptoms during coronavirus epidemics. $J$ Health Psychol [Epub ahead of print] 23 Aug 2020. Available from: https://doi.org/10. $1177 / 1359105320951620$.

4 Czeisler MÉ, Lane RI, Petrosky E, Wiley JF, Christensen A, Njai R, et al. Mental health, substance use, and suicidal ideation during the COVID-19 pandemic-United States, June 24-30, 2020. Morb Mortal Wkly Rep 2020; 69(32): 1049.

5 Reith TP. Burnout in United States healthcare professionals: a narrative review. Cureus 2018; 10(12): e3681.

6 Giusti EM, Pedroli E, D'Aniello GE, Badiale CS, Pietrabissa G, Manna C, et al. The psychological impact of the COVID-19 outbreak on health professionals: a cross-sectional study. Front Psychol 2020; 11: 1684.

7 Di Tella M, Romeo A, Benfante A, Castelli L. Mental health of healthcare workers during the COVID-19 pandemic in Italy. J Eval Clin Pract 2020; 26(6): 1583-7.

8 Maunder R, Hunter J, Vincent L, Bennett J, Peladeau N, Leszcz M, et al. The immediate psychological and occupational impact of the 2003 SARS outbreak in a teaching hospital. CMAJ 2003; 168(10): 1245-51.

9 Chua SE, Cheung V, Cheung C, MCAlonan GM, Wong JWS, Cheung EPT, et al. Psychological effects of the SARS outbreak in Hong Kong on high-risk health care workers. Can J Psychiatry 2004; 49(6): 391-3.

10 Lee SM, Kang WS, Cho A-R, Kim T, Park JK. Psychological impact of the 2015 MERS outbreak on hospital workers and quarantined hemodialysis patients. Compr Psychiatry 2018; 87: 123-7.

11 Que J, Le Shi JD, Liu J, Zhang L, Wu S, Gong Y, et al. Psychological impact of the COVID-19 pandemic on healthcare workers: a cross-sectional study in China. Gen Psychiatry 2020; 33(3): e100259.

12 Lai J, Ma S, Wang Y, Cai Z, Hu J, Wei N, et al. Factors associated with mental health outcomes among health care workers exposed to coronavirus disease 2019. JAMA Netw Open 2020; 3(3): e203976.

13 Sun D, Yang D, Li Y, Zhou J, Wang W, Wang Q, et al. Psychological impact of 2019 novel coronavirus (2019-nCoV) outbreak on health workers in China. Epidemiol Infect 2020; 148: e96.

14 Jahrami $\mathrm{H}$, BaHammam AS, AlGahtani $\mathrm{H}$, Ebrahim A, Faris M, AlEid K, et al. The examination of sleep quality for frontline healthcare 
workers during the outbreak of COVID-19. Sleep Breath [Epub ahead of print] 26 Jun 2020. Available from: https://doi.org/10.1007/s11325-02002135-9.

15 Italian Ministry of Health. COVID-19 Italy, Daily Situation Report. Italian Ministry of Health, 2020 (http://www.salute.gov.it/portale/nuovocoronavirus/dettaglio ContenutiNuovoCoronavirus.jsp?lingua=italiano\&id=5351\&area=nuovoCorona virus\&menu=vuoto)

16 Lazzerini M, Putoto G. COVID-19 in Italy: momentous decisions and many uncertainties. Lancet Glob Heal 2020; 8(5): e641-2.

17 Braquehais MD, Vargas-Cáceres S, Gómez-Durán E, Nieva G, Valero S, Casas M, et al. The impact of the COVID-19 pandemic on the mental health of healthcare professionals. QJM Int J Med 2020; 113: 613-7.

18 Weiss DS, Marmar CR. The Impact of Event Scale-Revised. In Assessing Psychological Trauma and PTSD (eds JP Wilson, TM Keane). Guilford Press, 1997.

19 Pietrantonio F, De Gennaro L, Di Paolo MC, Solano L. The Impact of Event Scale: validation of an Italian version. J Psychosom Res 2003; 55(4): 389-93.

20 Vanaken L, Scheveneels S, Belmans E, Hermans D. Validation of the impact of event scale with modifications for COVID-19 (IES-COVID19). Front Psychiatry 2020; 11: 738

21 Spielberger CD, Pedrabissi L, Santinello M. STAI: State-Trait Anxiety Inventory Forma Y: Manuale. [STAl: State-Trait Anxiety Inventory - Form Y: Handbook] Giunti Organizzazioni Speciali, 1989.

22 spielberger CD, Gorsuch RL, Lushene PR, Vagg PR, Jacobs GA. Manual for the State-Trait Spielberger Anxiety Inventory (Form Y). Consulting Psychologists Press, 1983.

23 Bergua V, Meillon C, Potvin O, Bouisson J, Le Goff M, Rouaud O, et al. The STAI-Y trait scale: psychometric properties and normative data from a large populationbased study of elderly people. Int Psychogeriatr 2012; 24(7): 1163.

24 Sirigatti S, Stefanile C, Menoni E. Per un adattamento italiano del Maslach Burnout Inventory (MBI). [Toward an Italian version of the Maslach Burnout Inventory (MBI)] Boll di Psicol App/ 1988; 187(188): 33-9.

25 Maslach $C$, Jackson SE. The Measurement of Experienced Burnout. J Organ Behav 1981; 2(2): 99-113.

26 Poghosyan L, Aiken LH, Sloane DM. Factor structure of the Maslach Burnout Inventory: an analysis of data from large scale cross-sectional surveys of nurses from eight countries. Int J Nurs Stud 2009; 46(7): 894-902.

27 Sirigatti S, Stefanile C. Adattamento e taratura per I'Italia [Adaptation and adjustment of the $\mathrm{MBI}$ for Italy]. In $\mathrm{MBI}$ Maslach Burnout Inventory Manuale (eds Maslach C, Jackson SE): 33-42. Firenze; Organizzazioni Speciali, 1993.

28 da Silva FCT, Neto MLR. Psychological effects caused by the COVID-19 pandemic in health professionals: a systematic review with meta-analysis. Prog Neuropsychopharmacol Biol Psychiatry 2020; 104: 110062

29 World Health Organization (WHO). Coronavirus Disease (COVID-19) Pandemic. WHO, 2020 (https://www.who.int/emergencies/diseases/novel-coronavirus2019).

30 Agostinelli A, La Torre G, Bevilacqua F, Chiaradia G, Specchia ML, Ricciardi W. Burnout in healthcare workers of a university teaching hospital in Rome Italy: a cross-sectional study. Ig Sanita Pubb/ 2008; 64(1): 41-52.
31 Bressi C, Manenti S, Porcellana M, Cevales D, Farina L, Felicioni I, et al. Haemato-oncology and burnout: an Italian survey. Br J Cancer 2008; 98(6): 1046-52.

32 Grassi L, Magnani K. Psychiatric morbidity and burnout in the medical profession: an Italian study of general practitioners and hospital physicians. Psychother Psychosom 2000; 69(6): 329-34.

33 Klersy C, Callegari A, Martinelli V, Vizzardi V, Navino C, Malberti F, et al. Burnout in health care providers of dialysis service in Northern Italy - a multicentre study. Nephrol Dial Transplant 2007; 22(8): 2283-90.

34 Deying HU, Yue K, Wengang LI, Qiuying H., Zhang X, Zhu LX, et al. Frontline nurses' burnout, anxiety, depression, and fear statuses and their associated factors during the COVID-19 outbreak in Wuhan, China: a large-scale crosssectional study. EClinicalMedicine 2020; 24: 100424.

35 Morgantini LA, Naha U, Wang H, Francavilla S, Acar Ö, Flores JM, et al. Factors contributing to healthcare professional burnout during the COVID-19 pandemic: a rapid turnaround global survey. PLOS One 2020; 15(9): e0238217.

36 Cai H, Tu B, Ma J, Chen L, Fu L, Jiang Y, et al. Psychological impact and coping strategies of frontline medical staff in Hunan between January and March 2020 during the outbreak of coronavirus disease 2019 (COVID-19) in Hubei, China. Med Sci Monit 2020; 26: e924171-1-16.

37 Huang L, Lin G, Tang L, Yu L, Zhou Z. Special Attention to Nurses' Protection during the COVID-19 Epidemic. Crit Care 2020; 24: 120

38 Purvanova RK, Muros JP. Gender differences in burnout: a meta-analysis. $J$ Vocat Behav 2010; 77(2): 168-85.

39 Ozbay F, Johnson DC, Dimoulas E, Morgan CA III, Charney D, Southwick S. Social support and resilience to stress: from neurobiology to clinical practice. Psychiatry (Edgmont) 2007; 4(5): 35.

40 Manczak EM, Skerrett KA, Gabriel LB, Ryan KA, Langenecker SA. Family support: a possible buffer against disruptive events for individuals with and without remitted depression. J Fam Psychol 2018; 32(7): 926.

41 Brooks SK, Dunn R, Amlôt R, Rubin GJ, Greenberg N. A systematic, thematic review of social and occupational factors associated with psychological outcomes in healthcare employees during an infectious disease outbreak. J Occup Environ Med 2018; 60(3): 248-57.

42 Bauchner H, Easley TJ. Health care heroes of the COVID-19 pandemic. JAMA 2020; 323(20): 2021

43 Sanfilippo F, Bignami $\mathrm{E}$, Lorini $\mathrm{FL}$, Astuto M. The importance of a "socially responsible" approach during COVID-19: the invisible heroes of science in Italy. Crit Care 2020; 24: 261

44 Strack J, Lopes P, Esteves F, Fernandez-Berrocal P. Must we suffer to succeed? $J$ Individ Differ 2017; 38(2): 113-24.

45 West CP, Dyrbye $L N$, Sinsky C, Trockel M, Tutty M, Nedelec L, et al. resilience and burnout among physicians and the general US working population. JAMA Netw Open 2020; 3(7): e209385. 